\title{
Electron trimer states in conventional superconductors
}

\author{
Ali Sanayei, ${ }^{1,2, *}$ Pascal Naidon, ${ }^{3, \dagger}$ and Ludwig Mathey ${ }^{1,2,4,+}$ \\ ${ }^{1}$ Zentrum für Optische Quantentechnologien, Universität Hamburg, Luruper Chaussee 149, D-22761 Hamburg, Germany \\ ${ }^{2}$ Institut für Laserphysik, Universität Hamburg, Luruper Chaussee 149, D-22761 Hamburg, Germany \\ ${ }^{3}$ RIKEN Nishina Center, RIKEN, Wakō 351-0198, Japan \\ ${ }^{4}$ The Hamburg Centre for Ultrafast Imaging, Luruper Chaussee 149, D-22761 Hamburg, Germany
}

(Received 3 September 2018; revised manuscript received 25 December 2019; accepted 11 February 2020; published 19 March 2020)

\begin{abstract}
We expand the Cooper problem by including a third electron in an otherwise empty band. We demonstrate the formation of a trimer state of two electrons above the Fermi sea and the third electron, for sufficiently strong interband attractive interaction. We show that the critical interaction strength is the lowest for small Fermi velocities, large masses of the additional electron, and large Debye energy. This trimer state competes with the formation of the two-electron Cooper pair, and can be created transiently via optical pumping.
\end{abstract}

DOI: 10.1103/PhysRevResearch.2.013341

\section{INTRODUCTION}

In a seminal paper, Ref. [1], Cooper showed that two electrons immersed in a Fermi sea form a bound state for arbitrarily weak attractive interactions. The Cooper problem assumes the dominance of the effective interaction induced by the electron-phonon interaction over the screened Coulomb potential [2-5]. It is modeled as constant in momentum space within a narrow energy range of the order of the Debye energy for the relative kinetic energy of the electrons. This simplified model distills the key features and energy scales of the full interaction induced by electron-phonon coupling that are relevant for the formation of the bound state and its properties. The existence of this bound state indicates that the noninteracting Fermi sea is unstable against pair formation, which suggests the emergence of a superconducting state. A more extensive theory of this state was provided by BCS theory [2-11], which elaborated on the essential ingredients that are necessary for the formation of conventional superconductors, pointed out by the Cooper problem and its solution.

Furthermore, and going beyond superconductivity, a Cooper problem can be formulated for any order parameter composed of two fermionic operators. For example, the weakcoupling limit of charge-density-wave and spin-density-wave orders can be formulated as the formation of an electron-hole pair. Here, the electron is restricted to occupy states outside the Fermi sea, and the hole is restricted to states inside the Fermi sea. Given this broad applicability of the Cooper

\footnotetext{
*asanayei@physnet.uni-hamburg.de

†pascal@ riken.jp

¥Imathey@ physnet.uni-hamburg.de
}

Published by the American Physical Society under the terms of the Creative Commons Attribution 4.0 International license. Further distribution of this work must maintain attribution to the author(s) and the published article's title, journal citation, and DOI. problem, it is of fundamental importance for condensedmatter physics.

While the solution of the Cooper problem is a twoparticle bound state, further down we will discuss the formation of three-particle bound states. As an example for three-body bound states, we mention Efimov trimers [12-15]. These trimers are formed of particles interacting via contact

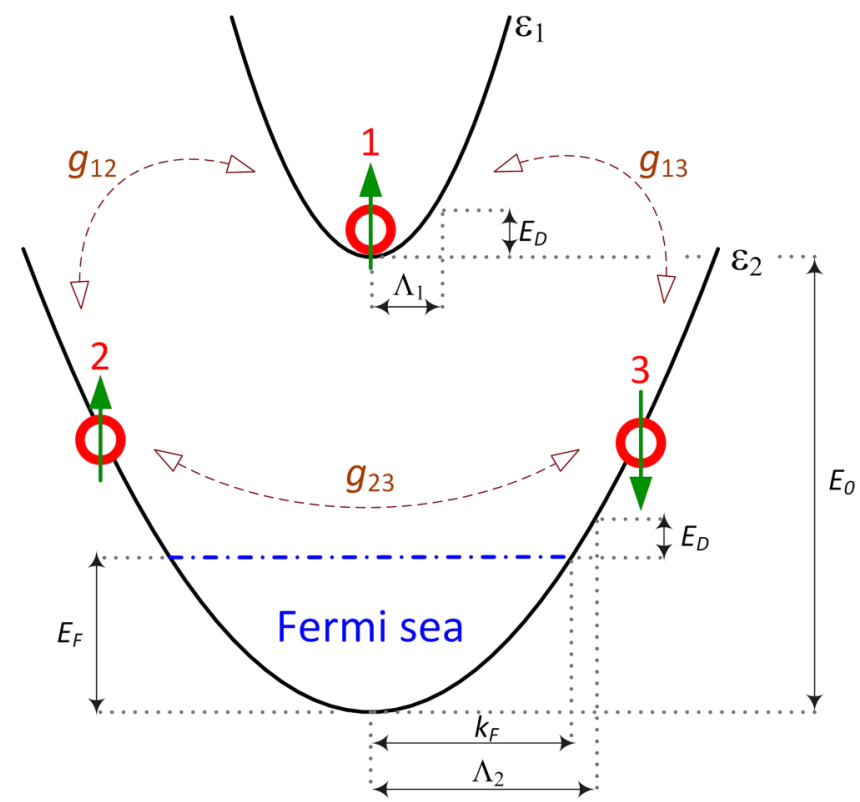

FIG. 1. The expanded Cooper problem consists of two electrons in a partially filled band with dispersion $\varepsilon_{2}$ and a third electron in an empty band with dispersion $\varepsilon_{1}$. The inert Fermi sea in the lower band has the Fermi energy $E_{F}$ and Fermi momentum $k_{F}$. The energy difference of the two bands is $E_{0}$. The three electrons interact attractively via the two-body interactions $g_{12}, g_{13}$, and $g_{23}$, with $g_{12}=g_{13}$. These interactions are cut off in momentum space by the cutoffs $\Lambda_{1}$ and $\Lambda_{2}$, whose magnitudes are chosen to fulfill $\varepsilon_{1}\left(\Lambda_{1}\right)=\varepsilon_{2}\left(\Lambda_{2}\right)-E_{F}=E_{D}$, where $E_{D}$ is the Debye energy. 


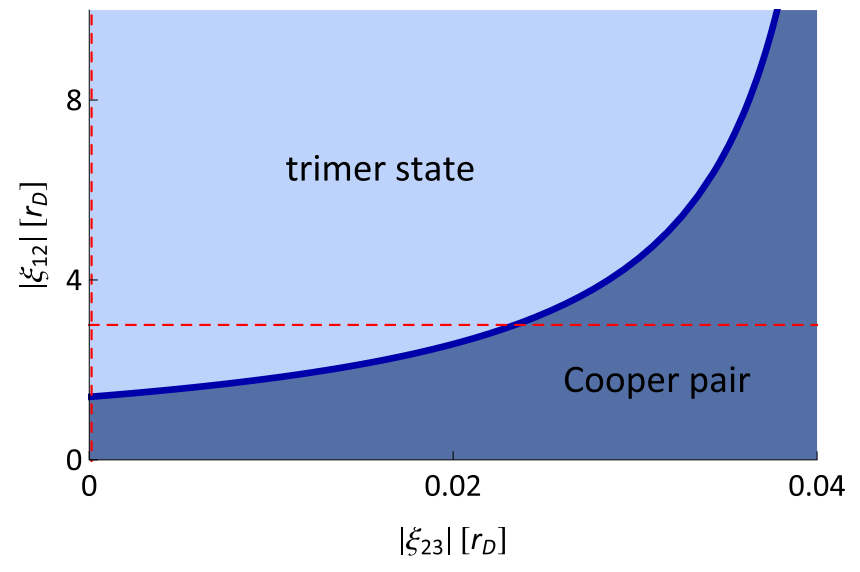

FIG. 2. Lowest energy state as a function of the interaction parameters $\left|\xi_{23}\right|$ and $\left|\xi_{12}\right|$, both in units of $r_{D}$. In this example, we choose $E_{D} / E_{F}=0.02, m_{2} / m_{1}=1$, and the cutoffs $\Lambda_{1}$ and $\Lambda_{2}$ according to Eq. (1). For sufficiently strong attractive interband interaction $\xi_{12}$, the trimer state has lower energy than the Cooper pair. The horizontal red dashed line is a cut at $\left|\xi_{12}\right|=3 r_{D}$, see Fig. 3, and the vertical red dashed line is a cut at $\left|\xi_{23}\right|=0$, see Fig. 4 .

interactions at interaction strengths that are not sufficient to support a two-particle bound state. Furthermore, in principle infinitely many three-body bound states are formed, which obey a scaling relation. In contrast to Cooper pairs, Efimov trimers are formed in vacuum. The Efimov effect has been experimentally observed in ultracold atomic gases [16-18] and helium beams experiments [19].

In this paper, we expand the Cooper problem by including a third electron, as depicted in Fig. 1, to demonstrate the formation of electron trimers in the presence of a Fermi sea. We assume that the additional electron, labeled " 1 ," is in an otherwise empty band with quadratic dispersion relation $\varepsilon_{1}$. Its spin state is arbitrarily depicted as spin-up. The two electrons "2" and "3" are restricted to be outside of an inert Fermi sea, where $k_{F}$ denotes the Fermi momentum and $E_{F}$ is the Fermi energy. For simplicity, the dispersion $\varepsilon_{2}$ of the lower band is assumed to be quadratic. We propose to realize this scenario by optically pumping electrons from a lower band into an unoccupied band, using current technology of pump-probe experiments [20-23]. This results in a low metastable electron density in the upper band. We assume that the effective interaction between the electrons is attractive, following the reasoning of the Cooper problem. We consider the interaction between two electrons to be a negative constant $g_{i j}$, with $i, j=1,2,3$ and $i \neq j$, for the incoming and outgoing momentum of particle $i$ smaller than a cutoff $\Lambda_{i}$, and zero otherwise. We choose the values of $\Lambda_{1}$ and $\Lambda_{2}=\Lambda_{3}$ such that

$$
E_{D}=\frac{\hbar^{2}}{2 m_{1}} \Lambda_{1}^{2}=\frac{\hbar^{2}}{2 m_{2}}\left(\Lambda_{2}^{2}-k_{F}^{2}\right),
$$

where $m_{i}$ is the effective mass of particle $i$ and $E_{D}$ is the Debye energy; see Fig. 1. For clarity, we allow for three different masses $m_{1}, m_{2}$, and $m_{3}$, but are primarily interested in the case $m_{3}=m_{2}$. A similar restriction will be placed on $g_{12}$ and $g_{13}$, which we choose to be equal throughout this paper. For a typical conventional superconductor we have $E_{D} \ll E_{F}$,

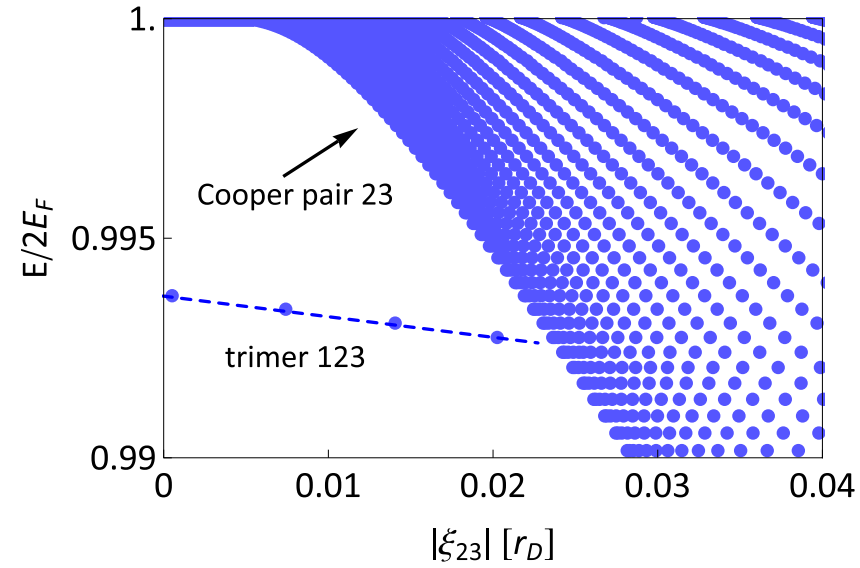

FIG. 3. Shifted energy $E=\mathcal{E}-E_{0}$ normalized by $2 E_{F}$ vs the interaction parameter $\left|\xi_{23}\right|$, in units of $r_{D}$, for $E_{D} / E_{F}=0.02, m_{2} / m_{1}=$ 1 , and $\xi_{12}=-3 r_{D}$, which corresponds to the horizontal red dashed line in Fig. 2. The lowest energy state for small values of $\left|\xi_{23}\right|$ is a trimer state. For sufficiently large values of $\left|\xi_{23}\right|$ the formation of Cooper pairs (dimer 23) will be dominant over a trimer state.

implying that $\Lambda_{1} \ll k_{F}$ and $\Lambda_{2}-k_{F} \ll k_{F}$, which we will use as small parameters further down. We define the length scale

$$
r_{D}=\left(\Lambda_{2}-k_{F}\right)^{-1} \approx \hbar \frac{v_{F}}{E_{D}},
$$

where $v_{F}=\hbar k_{F} / m_{2}$ is the Fermi velocity. We note that in the following we consider three-body states with vanishing total momentum.

\section{OVERVIEW AND MAIN RESULTS}

The main result of our analysis is shown in Fig. 2. We depict whether the lowest energy state is a Cooper pair state or a trimer state, as a function of the interaction parameters $\xi_{23}$ and $\xi_{12}=\xi_{13}$, where $\xi_{23}=2 \tilde{\mu} /\left(4 \pi \hbar^{2}\right) g_{23}$ and $\xi_{12}=2 \mu /\left(4 \pi \hbar^{2}\right) g_{12}$. The reduced masses are $1 / \tilde{\mu}=1 / m_{2}+$ $1 / m_{3}=2 / m_{2}$ and $1 / \mu=1 / m_{1}+1 / m_{2}$.

For $g_{12}=0$ we recover the result of the Cooper problem. For any value of $g_{23}<0$ the electrons in the lower band form a pair. As $g_{12}$ is set to a negative nonzero value, we show that the three electrons form a trimer state beyond a critical value of $g_{12}$, which increases in absolute magnitude as $\left|g_{23}\right|$ increases. This trimer formation also occurs for vanishing $g_{23}$. From the perspective of the electrons in the lower band, this can also be considered as a bound-state formation that is induced by a third electron in a higher band, i.e., a particle-induced bound state. As we show below, the magnitude of the critical value of $g_{12}$ for trimer formation is controlled by the ratio of the Fermi velocity, and the mass of the electron in the upper band and the Debye energy. If the mass of the electrons in the upper band is heavier, the critical value is reduced. Similarly, a smaller Fermi velocity and a larger Debye energy reduces the critical value. We note that for the typical parameter regime of conventional superconductors we find one trimer state only. However, we also give an example for a parameter regime in which more than one trimer state exists below.

For a pump-probe experiment, this result implies that a system that is initially either in a superconducting or a metallic 
state can be transformed into a Fermi liquid of electron trimers when electrons are pumped into a higher band, and the attractive interband interaction is sufficiently strong.

\section{ELECTRON TRIMER STATES}

To determine the bound states of the three-electron system we consider the Schrödinger equation in momentum space:

$$
\left(\frac{\hbar^{2} k_{1}^{2}}{2 m_{1}}+E_{0}+\frac{\hbar^{2} k_{2}^{2}}{2 m_{2}}+\frac{\hbar^{2} k_{3}^{2}}{2 m_{3}}+\hat{U}_{12}+\hat{U}_{13}+\hat{U}_{23}-\mathcal{E}\right) \psi=0
$$

where $\mathbf{k}_{1}, \mathbf{k}_{2}, \mathbf{k}_{3}$ are the electron momenta and $\psi=$ $\psi\left(\mathbf{k}_{1}, \mathbf{k}_{2}, \mathbf{k}_{3}\right)$ is the wave function. The total energy of the electron " 1 " is $\hbar^{2} k_{1}^{2} / 2 m_{1}+E_{0}$, where $E_{0}$ is the energy difference of the two bands; see Fig. 1. Here we define a shifted energy $E=\mathcal{E}-E_{0}$, where $\mathcal{E}$ is the eigenenergy [24]. The interaction $\hat{U}_{i j}$ between two electrons " $i$ " and " $j$ " is

$$
\hat{U}_{i j} \psi=g_{i j} \theta_{\Lambda_{i}}\left(\mathbf{k}_{i}\right) \theta_{\Lambda_{j}}\left(\mathbf{k}_{j}\right) \int \frac{d^{3} \mathbf{q}}{(2 \pi)^{3}} \theta_{\Lambda_{i}}\left(\mathbf{k}_{i}-\mathbf{q}\right) \theta_{\Lambda_{j}}\left(\mathbf{k}_{j}+\mathbf{q}\right) \psi,
$$

where $g_{i j}<0$ and $\mathbf{q}$ denotes the momentum transfer [25]; see Appendix A for the resulting operators $\hat{U}_{i j} \psi$. The cutoff function $\theta_{a, b}(\mathbf{k})$ is defined as

$$
\theta_{a, b}(\mathbf{k})= \begin{cases}1 & \text { for } a \leqslant|\mathbf{k}| \leqslant b, \\ 0 & \text { otherwise, }\end{cases}
$$

for two real numbers $0 \leqslant a<b$, and $\theta_{b}(\mathbf{k}) \equiv \theta_{0, b}(\mathbf{k})$. The inert Fermi sea demands the constraints $k_{2}>k_{F}$ and $k_{3}>k_{F}$ on the momenta of electrons " 2 " and " 3 ," respectively. We consider a singlet state for the electrons " 2 " and " 3 " in the following. This system is separable, see Appendix A, which results in a system of two coupled integral equations:

$$
\begin{aligned}
& {\left[\frac{1}{g_{12}}+\int \frac{d^{3} \mathbf{p}_{3}}{(2 \pi)^{3}} K_{1}\left(\mathbf{k}_{2}, \mathbf{p}_{3} ; E\right)\right] F_{2}\left(\mathbf{k}_{2}\right)} \\
& =-\theta_{k_{F}, \Lambda_{2}}\left(\mathbf{k}_{2}\right)\left[\int \frac{d^{3} \mathbf{p}_{3}}{(2 \pi)^{3}} K_{1}\left(\mathbf{k}_{2}, \mathbf{p}_{3} ; E\right) F_{2}\left(\mathbf{p}_{3}\right)\right. \\
& \left.\quad+\int \frac{d^{3} \mathbf{p}_{1}}{(2 \pi)^{3}} K_{2}\left(\mathbf{k}_{2}, \mathbf{p}_{1} ; E\right) F_{1}\left(\mathbf{p}_{1}\right)\right] \\
& {\left[\frac{1}{g_{23}}+\int \frac{d^{3} \mathbf{p}_{3}}{(2 \pi)^{3}} K_{3}\left(\mathbf{k}_{1}, \mathbf{p}_{3} ; E\right)\right] F_{1}\left(\mathbf{k}_{1}\right)} \\
& =-2 \theta_{\Lambda_{1}}\left(\mathbf{k}_{1}\right) \int \frac{d^{3} \mathbf{p}_{3}}{(2 \pi)^{3}} K_{3}\left(\mathbf{k}_{1}, \mathbf{p}_{3} ; E\right) F_{2}\left(\mathbf{p}_{3}\right),
\end{aligned}
$$

where $\mathbf{p}_{i}=\mathbf{k}_{i}-\mathbf{q}$, for $i=1,2$, and $\mathbf{p}_{3}=\mathbf{k}_{3}+\mathbf{q}$. The three integral kernels $K_{1}, K_{2}, K_{3}$ and the three functions $F_{1}, F_{2}$, $F_{3}$ are derived in Appendix A. Due to the singlet symmetry for electrons " 2 " and " 3 " we consider $F_{2}=F_{3}$. We assume $F_{i}(\mathbf{k})=F_{i}(k)$, implying $s$-wave symmetry of the state. We notice that in the absence of a Fermi sea and for a contact interaction, Eqs. (6) and (7) will reduce to the standard Skorniakov-Ter Martirosian equation [26] leading to the Efimov effect [27].

To determine whether the lowest energy state is a two-body or a three-body bound state, we use the small parameter
$E_{D} / E_{F}$ to approximate the full integral equation with an equation that relates $\xi_{23}, \xi_{12}$, and $E$; see Appendix B. We solve this equation numerically for eigenenergies $\mathcal{E}$ near the threshold energy $E_{\mathrm{thr}}=2 E_{F}+E_{0}=\hbar^{2} k_{F}^{2} / m_{2}+E_{0}$ [28] which results in Fig. 2, depicting a region where electrons " 2 " and " 3 " form a Cooper pair, and a second region where the three electrons form a trimer state. The intraband electrons " 2 " and " 3 " can form a Cooper pair for any attractive interaction $\xi_{23}$. The trimer state is only formed when the interband interaction $\xi_{12}$ is sufficiently strong. Trimer states of zero total momentum appear as discrete energy levels, whereas dimer states appear as continuum of states.

Next, we solve the full Eqs. (6) and (7) numerically to find the full spectrum and also to validate the analytical estimates. For that, we reduce the three-dimensional integrals over momentum to one-dimensional integrals over the absolute value of each momentum. We approximate the integrals by a sum over discrete values according to the Gauss-Legendre quadrature rule [29-31]. The continuous functions $F_{1}$ and $F_{2}$ are evaluated at these discrete momentum values. We therefore approximate Eqs. (6) and (7) with a discrete eigenvalue problem; see Appendix C.

We calculate the resulting eigenenergies $\mathcal{E}$ below the threshold energy $E_{\text {thr }}$. In Fig. 3 we show the shifted energy $E=\mathcal{E}-E_{0}$ normalized by $2 E_{F}$, for $E_{D} / E_{F}=0.02$ and $\xi_{12}=$ $-3 r_{D}$. For small values of $\left|\xi_{23}\right|$ the lowest energy state is a trimer state which appears as a single line of solutions. For larger values the lowest energy state is a Cooper pair which appears as the lowest energy state of a two-body bound-state continuum.

To estimate the critical value of $g_{12}$ for vanishing $g_{23}$ analytically, we recall that $F_{1} \propto g_{23}$ and $F_{2} \propto g_{12}$; cf. Eqs. (A6) and (A7). With this, Eqs. (6) and (7) reduce to

$$
\begin{aligned}
& \frac{4 \pi \hbar^{2}}{2 \mu g_{12}}+\frac{\tau}{\frac{\mu}{m_{1}} \pi k_{F}} \int_{k_{F}}^{\Lambda_{2}} d p_{3} p_{3} \\
& \times \ln \left(\frac{\left(1-\frac{\mu}{m_{1}}\right) p_{3}^{2}+\left(1-\frac{\mu}{m_{1}}\right) k_{F}^{2}+\frac{\mu}{m_{1}} \Lambda_{1}^{2}-\frac{2 \mu}{\hbar^{2}} E}{p_{3}^{2}-\frac{2 \mu}{m_{1}} k_{F} p_{3}+k_{F}^{2}-\frac{2 \mu}{\hbar^{2}} E}\right) \approx 0,
\end{aligned}
$$

where $\tau=1$ describes the system of three electrons and $\tau=$ $1 / 2$ corresponds to a system of two electrons, " 1 " and " 2 " (or "3"); see Appendix D. We evaluate the integral and expand to lowest order in $E_{D} / E_{F}$; see Appendices B and D. We solve for the interaction parameter $g_{12}$, then choose the threshold condition $\mathcal{E}=E_{\text {thr }}$ [28], which finally gives the critical value

$$
\left|g_{12}^{(\mathrm{c})}\right| \sim \frac{2 \pi^{2} \hbar^{2}}{m_{1}} r_{D} \approx \frac{2 \pi^{2} \hbar^{3}}{m_{1}} \frac{v_{F}}{E_{D}},
$$

where $v_{F}=\hbar k_{F} / m_{2}$; see Appendix D. This shows that a lower value of the critical interaction strength is achieved for heavier electrons in the upper band, for a smaller Fermi velocity, and a higher Debye energy.

An approximate analytical solution of Eq. (8), which describes both the trimer state and the lowest energy two-body bound state, is derived in Appendix D. These solutions are depicted as the red dashed and the red continuous line in Figs. 4(a)-4(c). For $m_{1} \gg m_{2}$ and $E_{D} \ll E_{F}$ we have $\left|\xi_{12}^{(\mathrm{c})}\right|=$ $2 \mu /\left(4 \pi \hbar^{2}\right)\left|g_{12}^{(\mathrm{c})}\right| \rightarrow 0^{+}$. In this case, a second analytical 

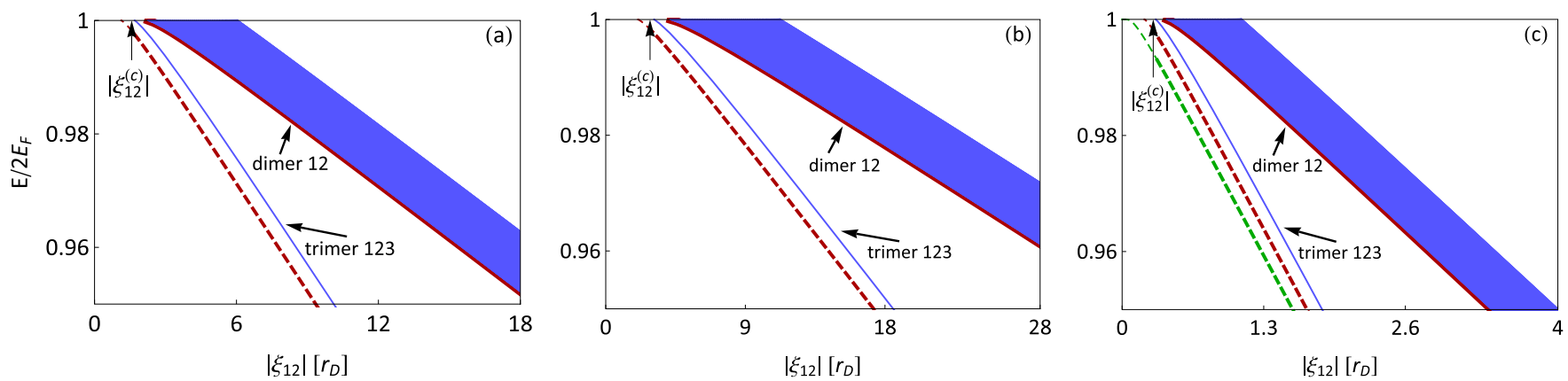

FIG. 4. Shifted energy $E=\mathcal{E}-E_{0}$ normalized by $2 E_{F}$ vs the interaction parameter $\left|\xi_{12}\right|$, in units of $r_{D}$, with $g_{23}=0$ and $E_{D} / E_{F}=0.02$, for (a) $m_{2} / m_{1}=1$, corresponding to the vertical red dashed line in Fig. 2, (b) $m_{2} / m_{1}=10$, and (c) $m_{2} / m_{1}=1 / 10$. In each panel, the single blue curve is the numerical solution of the lowest energy trimer state and the red dashed curve is the analytical approximation for the trimer state; see Appendix D. As $\left|\xi_{12}\right|$ increases, the first pair (dimer 12) appears as the lowest energy state of a two-body bound state continuum (blue dense curves) [32]. The red solid curve is the analytical approximation for the lowest energy pair (dimer 12); see Appendix D. The vertical arrow locates the critical value of the interband interaction parameter given by Eq. (9). In panel (c), the green dashed curve is the second analytical approximation (10), representing a good approximation for the asymptotic of the single blue curve as long as $m_{1} \gg m_{2}$.

approximate solution of Eq. (8) can be represented by

$$
E \approx 2 E_{F}+\frac{E_{D}}{1-\exp \left(\pi \frac{\mu}{m_{1}} \frac{r_{D}}{\left|\xi_{12}\right|}\right)} ;
$$

see Appendix D. This approximation is shown as a green dashed line in Fig. 4(c). For larger $m_{1} / m_{2}$, Eq. (10) becomes a better approximation. We compare these analytical results with the numerical results [32] for different parameter sets in Fig. 4. In Fig. 4(a) we use $m_{2}=m_{1}$ and $E_{D} / E_{F}=0.02$. In Fig. 4(b) we use $m_{2} / m_{1}=10$ and $E_{D} / E_{F}=0.02$. In Fig. 4(c) we use $m_{2} / m_{1}=1 / 10$ and $E_{D} / E_{F}=0.02$. We observe the formation of a trimer state, that is well approximated by the analytical approximation. In contrast to the Cooper problem where a two-body bound state originates at a vanishing coupling constant $[1,5,7]$, here, a trimer state emerges at the critical value $\xi_{12}^{(c)}$.

Finally, for $m_{2} \gg m_{1}$ and large values of the Debye energy that are comparable to the Fermi energy, $E_{D} \sim E_{F}$, we observe the formation of more than one trimer state. This is reminiscent of the Efimov effect, however, here the number of the trimers remains finite. Figure 5 shows the formation of two trimer states for $m_{2} / m_{1}=10$. Physical systems in this regime are superconductors like fullerides [33] or magnesium diboride [34].

\section{EXPERIMENTAL SIGNATURES}

The observation of trimer states in conventional superconductors can be realized in several scenarios. As a first experimental scenario, the trimer state can be detected as an excited state, as an in-gap resonance peak, $E_{0}>E_{F}$, while the electrons in the lower band are in a superconducting state, generically. Optically probing this state, see also Refs. [35,36], will result in the observation of a resonance at the trimer binding energy below the empty band. This resonance is broadened by the three-body nature of the bound state. We also propose to realize this effect by optically pumping electrons to an otherwise empty band [20]. Here, our study applies to a longlived metastable state created by optically pumping electrons to a higher band. For time scales below the relaxation rate from the upper band, the system will form a trimer state for the regime indicated in Fig. 2, giving rise to a Fermi liquid of trimers; see Fig. 6 in Appendix E.

As a second scenario, for trimer binding energies exceeding the energy difference of the bands, the trimer state is the ground state of the system and destabilizes the BCS state. Here, the upper band is lowered, $E_{0} \gtrsim E_{F}$, and touches the Fermi energy of the lower band, $E_{0} \sim E_{F}$; see Figs. 7(a) and 7(b) in Appendix F. One can also imagine that the upper band is lowered into the Fermi sea, $E_{0} \lesssim E_{F}$. Here, we assume that the upper band is very dilute; see Fig. 7(c) in Appendix F. Because of this, new electron pockets will appear. Our prediction is now that trimer formation is possible as the ground state, if the Fermi sea is tuned to formation of the electron pockets. For $g_{23}=0$, the formation of a trimer liquid is achieved for any density of fermions in the upper band, if $g_{12}$ and $g_{13}$ are above the critical interaction strength. If $g_{23}$ induces a BCS state in the lower band, the energy reduction of the formation

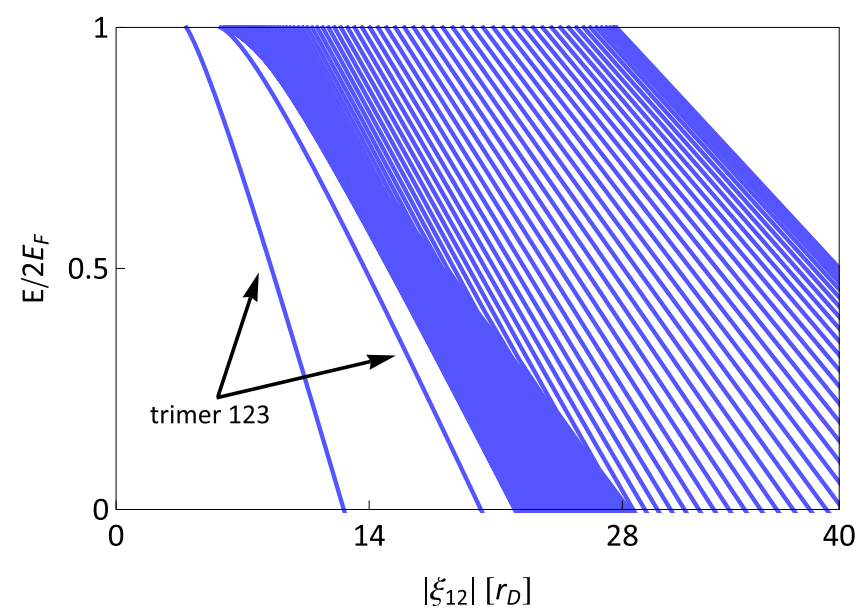

FIG. 5. The resulting three-body shifted energy $E=\mathcal{E}-E_{0}$ normalized by $2 E_{F}$ vs the interaction parameter $\left|\xi_{12}\right|$, in units of $r_{D}$, with $g_{23}=0$ and $E_{D} \sim E_{F}$, for $m_{2} / m_{1}=10$. Here, we see the formation of two trimer states that are shown by two single blue curves. The dense curves show the two-body bound-state continuum [32]. When $m_{2} \gg m_{1}$, we can see the formation of more than one trimer state. 
of a trimer liquid has to be compared to the energy of the BCS state. For sufficiently high density of electrons in the upper band, the trimer state competes with the BCS order.

Finally, to realize our prediction of forming more than one trimer state, see Fig. 5, BCS-like superconductors, e.g., fullerides or magnesium diboride, can be used, where $m_{2} \gg$ $m_{1}$ and the Debye energy is comparable to the Fermi energy. For high-temperature superconductors similar considerations for sufficiently strong interband attractive interactions might apply. Here, the formation of a trimer state might support pairing enhancement $[37,38]$.

\section{CONCLUSIONS}

In conclusion, we have demonstrated the formation of a trimer state of electrons in a conventional superconductor, in which an additional electron occupies a higher band. We show this by expanding the Cooper problem of two attractively interacting electrons by adding an additional electron that also interacts attractively with the other electrons. The trimer formation sets in beyond a critical interband interaction strength, for which we give an analytical estimate. This demonstrates an instability of the optically pumped BCS state. Out of the initial superconducting or metallic state, a transient state of a Fermi liquid of electron trimers can be formed.

As mentioned above, the analog of the Cooper problem can also be formulated for orders such as spin-density-wave or charge-density-wave orders, as a two-body problem of an electron and a hole. We emphasize that the analysis of this paper can be extended to any order that is described by a twofermion order parameter, and predicts three-fermion bound states for all these orders for the corresponding parameter regimes.

\section{ACKNOWLEDGMENTS}

A.S. and L.M. acknowledge support from the Deutsche Forschungsgemeinschaft through Program No. SFB 925, and also The Hamburg Centre for Ultrafast Imaging as well as the Hamburg Cluster of Excellence Advanced Imaging of Matter (AIM). P.N. acknowledges support from the RIKEN Incentive Research Projects and JSPS Grants in-Aid for Scientific Research on Innovative Areas (No. JP18H05407).

\section{APPENDIX A: DERIVATION OF THE SYSTEM OF TWO COUPLED INTEGRAL EQUATIONS (6) AND (7)}

We apply the interaction operators $\hat{U}_{i j}$, given by Eq. (4), on the wave function $\psi=\psi\left(\mathbf{k}_{1}, \mathbf{k}_{2}, \mathbf{k}_{3}\right)$ governing the three-electron system in momentum space, and write the
Schrödinger Eq. (3) as follows:

$$
\left(\frac{\hbar^{2} k_{1}^{2}}{2 m_{1}}+\frac{\hbar^{2} k_{2}^{2}}{2 m_{2}}+\frac{\hbar^{2} k_{3}^{2}}{2 m_{3}}-E\right) \psi=-\left(\hat{U}_{12}+\hat{U}_{13}+\hat{U}_{23}\right) \psi,
$$

where

$$
\begin{aligned}
\hat{U}_{12} \psi= & g_{12} \theta_{\Lambda_{1}}\left(\mathbf{k}_{1}\right) \theta_{\Lambda_{2}}\left(\mathbf{k}_{2}\right) \int \frac{d^{3} \mathbf{q}}{(2 \pi)^{3}} \theta_{\Lambda_{1}}\left(\mathbf{k}_{1}-\mathbf{q}\right) \\
& \times \theta_{\Lambda_{2}}\left(\mathbf{k}_{2}+\mathbf{q}\right) \psi\left(\mathbf{k}_{1}-\mathbf{q}, \mathbf{k}_{2}+\mathbf{q}, \mathbf{k}_{3}\right), \\
\hat{U}_{13} \psi= & g_{13} \theta_{\Lambda_{1}}\left(\mathbf{k}_{1}\right) \theta_{\Lambda_{3}}\left(\mathbf{k}_{3}\right) \int \frac{d^{3} \mathbf{q}}{(2 \pi)^{3}} \theta_{\Lambda_{1}}\left(\mathbf{k}_{1}-\mathbf{q}\right) \\
& \times \theta_{\Lambda_{3}}\left(\mathbf{k}_{3}+\mathbf{q}\right) \psi\left(\mathbf{k}_{1}-\mathbf{q}, \mathbf{k}_{2}, \mathbf{k}_{3}+\mathbf{q}\right), \\
\hat{U}_{23} \psi= & g_{23} \theta_{\Lambda_{2}}\left(\mathbf{k}_{2}\right) \theta_{\Lambda_{3}}\left(\mathbf{k}_{3}\right) \int \frac{d^{3} \mathbf{q}}{(2 \pi)^{3}} \theta_{\Lambda_{2}}\left(\mathbf{k}_{2}-\mathbf{q}\right) \\
& \times \theta_{\Lambda_{3}}\left(\mathbf{k}_{3}+\mathbf{q}\right) \psi\left(\mathbf{k}_{1}, \mathbf{k}_{2}-\mathbf{q}, \mathbf{k}_{3}+\mathbf{q}\right) .
\end{aligned}
$$

Here, $E=\mathcal{E}-E_{0}$ is the shifted energy, where $\mathcal{E}$ denotes the eigenenergy and $E_{0}$ is the energy difference of the two bands; see Fig. 1. The function $\theta_{a, b}(\mathbf{k})$ is defined by Eq. (5) and $\theta_{b}(\mathbf{k}) \equiv \theta_{0, b}(\mathbf{k})$.

Next, we introduce three variables $\tilde{\mathbf{p}}_{i} \equiv \mathbf{q}+\mathbf{k}_{i}$, with $i=$ $1,2,3$, and assume the zero total momentum of the system, $\psi\left(\mathbf{k}_{1}, \mathbf{k}_{2}, \mathbf{k}_{3}\right)=\psi\left(\mathbf{k}_{2}, \mathbf{k}_{3}\right) \delta^{(3)}\left(\mathbf{k}_{1}+\mathbf{k}_{2}+\mathbf{k}_{3}\right)$, where $\delta^{(3)}$ is the three-dimensional Dirac delta function. We rewrite Eq. (A1) in the following form:

$$
\begin{aligned}
& \left(\frac{\hbar^{2}\left(\mathbf{k}_{2}+\mathbf{k}_{3}\right)^{2}}{2 m_{1}}+\frac{\hbar^{2} k_{2}^{2}}{2 m_{2}}+\frac{\hbar^{2} k_{3}^{2}}{2 m_{3}}-E\right) \psi\left(\mathbf{k}_{2}, \mathbf{k}_{3}\right) \\
& =-\theta_{\Lambda_{1}}\left(-\mathbf{k}_{2}-\mathbf{k}_{3}\right) \theta_{\Lambda_{2}}\left(\mathbf{k}_{2}\right) F_{3}\left(\mathbf{k}_{3}\right)-\theta_{\Lambda_{1}}\left(-\mathbf{k}_{2}-\mathbf{k}_{3}\right) \\
& \quad \times \theta_{\Lambda_{3}}\left(\mathbf{k}_{3}\right) F_{2}\left(\mathbf{k}_{2}\right)-\theta_{\Lambda_{2}}\left(\mathbf{k}_{2}\right) \theta_{\Lambda_{3}}\left(\mathbf{k}_{3}\right) F_{1}\left(-\mathbf{k}_{2}-\mathbf{k}_{3}\right),
\end{aligned}
$$

where the functions $F_{1}, F_{2}$, and $F_{3}$ are defined as

$$
\begin{aligned}
& F_{1}\left(\mathbf{k}_{1}\right)= g_{23} \int \frac{d^{3} \tilde{\mathbf{p}}_{3}}{(2 \pi)^{3}} \theta_{\Lambda_{2}}\left(-\mathbf{k}_{1}-\tilde{\mathbf{p}}_{3}\right) \theta_{\Lambda_{3}}\left(\tilde{\mathbf{p}}_{3}\right) \\
& \times \psi\left(-\mathbf{k}_{1}-\tilde{\mathbf{p}}_{3}, \tilde{\mathbf{p}}_{3}\right), \\
& F_{2}\left(\mathbf{k}_{2}\right)=g_{13} \int \frac{d^{3} \tilde{\mathbf{p}}_{3}}{(2 \pi)^{3}} \theta_{\Lambda_{1}}\left(-\mathbf{k}_{2}-\tilde{\mathbf{p}}_{3}\right) \theta_{\Lambda_{3}}\left(\tilde{\mathbf{p}}_{3}\right) \psi\left(\mathbf{k}_{2}, \tilde{\mathbf{p}}_{3}\right),
\end{aligned}
$$

$$
F_{3}\left(\mathbf{k}_{3}\right)=g_{12} \int \frac{d^{3} \tilde{\mathbf{p}}_{2}}{(2 \pi)^{3}} \theta_{\Lambda_{1}}\left(-\mathbf{k}_{3}-\tilde{\mathbf{p}}_{2}\right) \theta_{\Lambda_{2}}\left(\tilde{\mathbf{p}}_{2}\right) \psi\left(\tilde{\mathbf{p}}_{2}, \mathbf{k}_{3}\right) .
$$

Equation (A5) provides now an ansatz for the wave function $\psi\left(\mathbf{k}_{2}, \mathbf{k}_{3}\right)$ :

$$
\psi\left(\mathbf{k}_{2}, \mathbf{k}_{3}\right)=-\frac{\theta_{\Lambda_{1}}\left(-\mathbf{k}_{2}-\mathbf{k}_{3}\right) \theta_{\Lambda_{2}}\left(\mathbf{k}_{2}\right) F_{3}\left(\mathbf{k}_{3}\right)+\theta_{\Lambda_{1}}\left(-\mathbf{k}_{2}-\mathbf{k}_{3}\right) \theta_{\Lambda_{3}}\left(\mathbf{k}_{3}\right) F_{2}\left(\mathbf{k}_{2}\right)+\theta_{\Lambda_{2}}\left(\mathbf{k}_{2}\right) \theta_{\Lambda_{3}}\left(\mathbf{k}_{3}\right) F_{1}\left(-\mathbf{k}_{2}-\mathbf{k}_{3}\right)}{\frac{\hbar^{2}\left(\mathbf{k}_{2}+\mathbf{k}_{3}\right)^{2}}{2 m_{1}}+\frac{\hbar^{2} k_{2}^{2}}{2 m_{2}}+\frac{\hbar^{2} k_{3}^{2}}{2 m_{3}}-E} .
$$

If electrons " 2 " and " 3 " are in a spin singlet state and $g_{12}=g_{13}$, then $F_{3}=F_{2}$. We also assume $m_{3}=m_{2}$ and take into account the Fermi sea condition by $k_{2}>k_{F}$ and $k_{3}>k_{F}$. Next, we introduce the variables $\mathbf{p}_{1} \equiv-\mathbf{k}_{2}-\tilde{\mathbf{p}}_{3}$ and 
$\mathbf{p}_{2} \equiv-\mathbf{k}_{1}-\tilde{\mathbf{p}}_{3}$, with $\mathbf{p}_{3} \equiv \tilde{\mathbf{p}}_{3}$. We also assume $\Lambda_{3}=\Lambda_{2}$. The functions $F_{1}$ and $F_{2}$ now read

$$
\begin{gathered}
F_{1}\left(\mathbf{k}_{1}\right)=g_{23} \int \frac{d^{3} \mathbf{p}_{3}}{(2 \pi)^{3}} \theta_{k_{F}, \Lambda_{2}}\left(-\mathbf{k}_{1}-\mathbf{p}_{3}\right) \theta_{k_{F}, \Lambda_{2}}\left(\mathbf{p}_{3}\right) \\
\times \psi\left(-\mathbf{k}_{1}-\mathbf{p}_{3}, \mathbf{p}_{3}\right), \\
F_{2}\left(\mathbf{k}_{2}\right)=g_{12} \int \frac{d^{3} \mathbf{p}_{3}}{(2 \pi)^{3}} \theta_{\Lambda_{1}}\left(-\mathbf{k}_{2}-\mathbf{p}_{3}\right) \theta_{k_{F}, \Lambda_{2}}\left(\mathbf{p}_{3}\right) \psi\left(\mathbf{k}_{2}, \mathbf{p}_{3}\right) .
\end{gathered}
$$

We insert the ansatz (A9) into Eqs. (A10) and (A11), and arrive at a system of two coupled integral Eqs. (6) and (7), where the three kernels $K_{1}, K_{2}$, and $K_{3}$ are

$$
\begin{gathered}
K_{1}\left(\mathbf{k}_{2}, \mathbf{p}_{3} ; E\right)=\frac{\theta_{\Lambda_{1}}\left(-\mathbf{k}_{2}-\mathbf{p}_{3}\right) \theta_{k_{F}, \Lambda_{2}}\left(\mathbf{p}_{3}\right)}{\frac{\hbar^{2}\left(\mathbf{k}_{2}+\mathbf{p}_{3}\right)^{2}}{2 m_{1}}+\frac{\hbar^{2} k_{2}^{2}}{2 m_{2}}+\frac{\hbar^{2} p_{3}^{2}}{2 m_{2}}-E}, \\
K_{2}\left(\mathbf{k}_{2}, \mathbf{p}_{1} ; E\right)=\frac{\theta_{\Lambda_{1}}\left(\mathbf{p}_{1}\right) \theta_{k_{F}, \Lambda_{2}}\left(-\mathbf{p}_{1}-\mathbf{k}_{2}\right)}{\frac{\hbar^{2} p_{1}^{2}}{2 m_{1}}+\frac{\hbar^{2} k_{2}^{2}}{2 m_{2}}+\frac{\hbar^{2}\left(\mathbf{p}_{1}+\mathbf{k}_{2}\right)^{2}}{2 m_{2}}-E}, \\
K_{3}\left(\mathbf{k}_{1}, \mathbf{p}_{3} ; E\right)=\frac{\theta_{k_{F}, \Lambda_{2}}\left(-\mathbf{k}_{1}-\mathbf{p}_{3}\right) \theta_{k_{F}, \Lambda_{2}}\left(\mathbf{p}_{3}\right)}{\frac{\hbar^{2} k_{1}^{2}}{2 m_{1}}+\frac{\hbar^{2}\left(\mathbf{k}_{1}+\mathbf{p}_{3}\right)^{2}}{2 m_{2}}+\frac{\hbar^{2} p_{3}^{2}}{2 m_{2}}-E} .
\end{gathered}
$$

\section{APPENDIX B: OVERALL BEHAVIOR OF THE THREE-ELECTRON SYSTEM}

As mentioned, we choose the values of the cutoffs $\Lambda_{1}$ and $\Lambda_{2}$ according to relation (1). For a typical conventional superconductor we have $E_{D} \ll E_{F}$, implying that $\Lambda_{1} \ll k_{F}$ and $\Lambda_{2}-k_{F} \ll k_{F}$. We recall that $0<k_{1}<\Lambda_{1}$ and $k_{F}<$ $k_{2}<\Lambda_{2}$. We thus make a first approximation such that $k_{1} \sim 0$ and $k_{2} \sim k_{F}$. In addition, because the integral variable $p_{3}$ is varying within the interval $\left(k_{F}, \Lambda_{2}\right)$, and also $\Lambda_{2}-k_{F} \ll$ $k_{F}$, we make a second approximation in this interval and assume that the two functions $F_{2}\left(k_{2}\right)$ and $F_{2}\left(p_{3}\right)$ remain constant, $F_{2}\left(k_{F}\right)$. We rewrite the system of Eqs. (6) and (7) as follows:

$$
\left\{\begin{array}{l}
\Omega_{1}\left(E, g_{12} ; \tau\right) F_{2}\left(k_{F}\right)+\Omega_{2}(E) F_{1}(0) \approx 0, \\
\Omega_{3}(E) F_{2}\left(k_{F}\right)+\Omega_{4}\left(E, g_{23}\right) F_{1}(0) \approx 0 .
\end{array}\right.
$$

We recall that $\tau=1$ describes the system of three electrons and $\tau=1 / 2$ corresponds to a system of two electrons " 1 " and " 2 " (or "3"). We calculate the function $\Omega_{1}\left(E, g_{12} ; \tau\right)$ to be

$$
\Omega_{1}\left(E, g_{12} ; \tau\right)=\frac{4 \pi \hbar^{2}}{2 \mu g_{12}}+\tilde{\Omega}_{1}(E ; \tau),
$$

where

$$
\begin{aligned}
\tilde{\Omega}_{1}(E ; \tau)= & \frac{\tau}{\frac{\mu}{m_{1}} \pi k_{F}} \int_{k_{F}}^{\Lambda_{2}} d p_{3} p_{3} \ln \left(\frac{\left(1-\frac{\mu}{m_{1}}\right) p_{3}^{2}+\left(1-\frac{\mu}{m_{1}}\right) k_{F}^{2}+\frac{\mu}{m_{1}} \Lambda_{1}^{2}-\frac{2 \mu}{\hbar^{2}} E}{p_{3}^{2}-\frac{2 \mu}{m_{1}} k_{F} p_{3}+k_{F}^{2}-\frac{2 \mu}{\hbar^{2}} E}\right) \\
= & \frac{\tau}{\pi}\left(\Lambda_{2}-k_{F}\right)+\frac{2 \tau}{\pi} \sqrt{\eta(E)}\left[\arctan \left(\frac{\frac{\mu}{m_{1}} k_{F}-\Lambda_{2}}{\sqrt{\eta(E)}}\right)-\arctan \left(\frac{\left(\frac{\mu}{m_{1}}-1\right) k_{F}}{\sqrt{\eta(E)}}\right)\right] \\
& +\frac{\tau \Lambda_{2}^{2}}{\frac{2 \mu}{m_{1}} \pi k_{F}} \ln \left(\frac{-\rho(E)+\left(1-\frac{\mu}{m_{1}}\right) \Lambda_{2}^{2}}{\chi(E)}\right)-\frac{\tau k_{F}}{\frac{2 \mu}{m_{1}} \pi} \ln \left(\frac{\rho(E)+\left(\frac{\mu}{m_{1}}-1\right) k_{F}^{2}}{\rho(E)+\left(\frac{\mu}{m_{1}}-1\right) k_{F}^{2}+\frac{\mu}{m_{1}} \Lambda_{1}^{2}}\right)+\frac{\tau \rho(E)}{\frac{2 \mu}{m_{1}}\left(\frac{\mu}{m_{1}}-1\right) \pi k_{F}} \\
& \times \ln \left(\frac{\rho(E)+\left(\frac{\mu}{m_{1}}-1\right) \Lambda_{2}^{2}}{\rho(E)+\left(\frac{\mu}{m_{1}}-1\right) k_{F}^{2}}\right)-\frac{\tau\left[\eta(E)-\left(\frac{\mu}{m_{1}}\right)^{2} k_{F}^{2}\right]}{\frac{2 \mu}{m_{1}} \pi k_{F}} \ln \left(\frac{-\rho(E)+\left(1-\frac{\mu}{m_{1}}\right) k_{F}^{2}-\frac{\mu}{m_{1}} \Lambda_{1}^{2}}{-}\right) .
\end{aligned}
$$

Here, $\eta(E)=\left[1-\left(\frac{\mu}{m_{1}}\right)^{2}\right] k_{F}^{2}-\frac{2 \mu}{\hbar^{2}} E, \rho(E)=\left(\frac{\mu}{m_{1}}-1\right) k_{F}^{2}-\frac{\mu}{m_{1}} \Lambda_{1}^{2}+\frac{2 \mu}{\hbar^{2}} E, \quad \chi(E)=k_{F}^{2}-\frac{2 \mu}{m_{1}} k_{F} \Lambda_{2}+\Lambda_{2}^{2}-\frac{2 \mu}{\hbar^{2}} E$, and $1 / \mu=$ $1 / m_{1}+1 / m_{2}$. For $\Lambda_{1} \ll k_{F}$ and $\Lambda_{2}-k_{F} \ll k_{F}$, we obtain

$$
\begin{aligned}
& \Omega_{2}(E)=\frac{1}{\frac{2 \mu}{m_{2}} \pi k_{F}} \int_{0}^{\Lambda_{1}} d p_{1} p_{1} \ln \left(\frac{p_{1}^{2}+\frac{2 \mu}{m_{2}} k_{F} p_{1}+k_{F}^{2}-\frac{2 \mu}{\hbar^{2}} E}{p_{1}^{2}-\frac{2 \mu}{m_{2}} k_{F} p_{1}+k_{F}^{2}-\frac{2 \mu}{\hbar^{2}} E}\right) \approx \frac{2}{3 \pi} \frac{\Lambda_{1}^{3}}{k_{F}^{2}-\frac{2 \mu}{\hbar^{2}} E}, \\
& \Omega_{3}(E)=\frac{2}{\frac{2 \tilde{\mu}}{m_{2}} \pi k_{F}} \int_{k_{F}}^{\Lambda_{2}} d p_{3} \frac{p_{3}}{k_{1}} \ln \left(\frac{\frac{2 \tilde{\mu}}{m_{2}} p_{3}^{2}+\frac{2 \tilde{\mu}}{m_{2}} k_{1} p_{3}+\frac{\tilde{\mu}}{\mu} k_{1}^{2}-\frac{\tilde{\mu}}{\mu} \frac{2 \mu}{\hbar^{2}} E}{\frac{2 \tilde{\mu}}{m_{2}} p_{3}^{2}-\frac{2 \tilde{\mu}}{m_{2}} k_{1} p_{3}+\frac{\tilde{\mu}}{\mu} k_{1}^{2}-\frac{\tilde{\mu}}{\mu} \frac{2 \mu}{\hbar^{2}} E}\right) \sim \frac{4}{\pi} \int_{k_{F}}^{\Lambda_{2}} d p_{3} \frac{p_{3}^{2}}{p_{3}^{2}-\frac{\tilde{\mu}}{\mu} \frac{2 \mu}{\hbar^{2}} E} \\
& \approx \frac{4}{\pi}\left[\Lambda_{2}-k_{F}+\frac{k_{F}}{2} \ln \left(\frac{2 k_{F}\left(\Lambda_{2}-k_{F}\right)}{k_{F}^{2}-\frac{\tilde{\mu}}{\mu} \frac{2 \mu}{\hbar^{2}} E}\right)\right] \text {, } \\
& \Omega_{4}\left(E, g_{23}\right)=\frac{4 \pi \hbar^{2}}{2 \tilde{\mu} g_{23}}+\frac{1}{2} \Omega_{3}(E),
\end{aligned}
$$

where $1 / \tilde{\mu}=1 / m_{2}+1 / m_{3}=2 / m_{2}$. 
In order that Eq. (B1) possesses nontrivial solutions, it is required that

$$
\Omega_{1}\left(E, g_{12} ; \tau=1\right) \Omega_{4}\left(E, g_{23}\right)-\Omega_{2}(E) \Omega_{3}(E)=0,
$$

which gives rise to a relation between $g_{12}$ and $g_{23}$ through $E$. Figure 2 shows the result for $E=\mathcal{E}-E_{0} \approx 2 E_{F}$, where $E_{F}=\hbar^{2} k_{F}^{2} / 2 m_{2}$ is the Fermi energy and $k_{F}$ is the Fermi momentum.

\section{APPENDIX C: NUMERICAL SOLUTIONS OF THE SYSTEM OF TWO COUPLED INTEGRAL EQUATIONS (6) AND (7)}

As mentioned, we assume $F_{i}(\mathbf{k})=F_{i}(k)$, implying that we only consider the isotropic solutions of Eqs. (6) and (7). To solve Eqs. (6) and (7) numerically we therefore replace the three-dimensional integrals over momentum by one-dimensional integrals over absolute values of each momentum. We discretize each integral range such that the grid points $\left\{x_{j}\right\}, j=1,2, \ldots, N$, are the set of zeros of the Legendre polynomials $P_{N}(x)$. We approximate the integrals by a truncated sum weighted by $w_{j}$ :

$$
w_{j}=\frac{2}{\left(1-x_{j}^{2}\right)\left[P_{N}^{\prime}\left(x_{j}\right)\right]^{2}},
$$

where $P_{N}^{\prime}(x)=d P_{N}(x) / d x[30,31]$. This choice, which is the so-called Gauss-Legendre quadrature rule, scales the range of integration from a given real interval $(a, b)$ to $(-1,1)$, and has order of accuracy exactly $2 N-1$, which is the highest accuracy among the other quadrature choices [29].

We apply the Gauss-Legendre quadrature rule on each integral and construct a matrix equation analog to each integral equation. For given values of $E$ below $2 E_{F}$ [28] we calculate the eigenvalues, which then provide the corresponding values of the interaction parameter. The functions $F_{1}$ and $F_{2}$ are also obtained as the eigenvectors of the matrix equations. We notice that, due to the truncation on each sum, the two-body continuum revealed in Figs. 3-5 has a finite range.

\section{APPENDIX D: DERIVATION AND SOLUTION OF EQUATION (8) AND DERIVATION OF EQUATIONS (9) AND (10)}

To derive Eq. (8), recall that for vanishing $g_{23}$ we have $F_{1}=0$, and therefore Eq. (7) will have no effect anymore. As discussed in Appendix B, for a conventional superconductor we make an approximation such that $k_{1} \sim 0$ and $k_{2} \sim k_{F}$. The system of three electrons is then described by Eq. (8) for $\tau=1$. The system of two electrons " 1 " and " 2 " (or " 3 ") will be described by the same equation when $\tau=1 / 2$.

Equation (8) for $\tau=1$ is then solved by

$$
\Omega_{1}\left(E, g_{12} ; \tau=1\right)=0,
$$

where the function $\Omega_{1}\left(E, g_{12} ; \tau\right)$ was calculated in Appendix B; see Eq. (B2). Equation (D1) provides now a relation between the interaction parameter $g_{12}$ and the shifted energy $E$; see red dashed curves in Fig. 4.

For the system of two electrons "1" and "2" (or " 3 ") we set $\tau=1 / 2$ and calculate the integral by the same argument. The

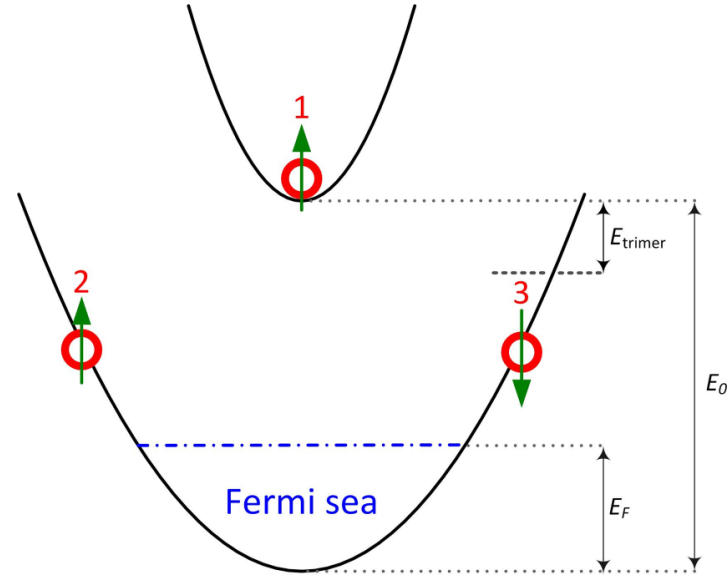

FIG. 6. Sketch of the first scenario for the experimental signature of the trimer states. The trimer state can be detected as an excited state, as an in-gap resonance peak, by either optically probing the state or optically pumping electrons from the lower band. Here, $E_{0}>$ $E_{F}$ and $E_{\text {trimer }}$ is the trimer binding energy.

lowest energy two-body bound state is obtained by solving

$$
\Omega_{1}\left(E, g_{12} ; \tau=1 / 2\right)=0 ;
$$

see red solid curves in Fig. 4.

To calculate the onset of the trimer state analytically, we expand Eq. (D1) for $\Lambda_{2}-k_{F} \ll k_{F}$ and $\Lambda_{1} \ll k_{F}$ at the shifted energy $E \approx 2 E_{F}=\hbar^{2} k_{F}^{2} / m_{2}$ [28], and solve the leading order for the interaction parameter $g_{12}^{(\mathrm{c})} \equiv g_{12}\left(E=2 E_{F}\right)$, which results in Eq. (9).

To derive Eq. (10), we notice that the onset of the trimer state for $m_{1} \gg m_{2}$ leads to the origin, $\left|\xi_{12}^{(\mathrm{c})}\right|=$ $2 \mu /\left(4 \pi \hbar^{2}\right)\left|g_{12}^{(\mathrm{c})}\right| \rightarrow 0^{+}$. In this case, to find the asymptotic of the trimer state we solve the integral appearing in Eq. (B2) by changing a variable $X \equiv p_{3} / k_{F}$. The integral bounds will then be 1 and $\Lambda_{2} / k_{F}$. For a conventional superconductor the upper bound $\Lambda_{2} / k_{F}$ is very close to the lower bound; therefore, we calculate the integral by making the leading order of the integrand when $X \rightarrow 1$. We solve the result for $E$ and arrive at Eq. (10); see green dashed curve in Fig. 4(c) of the paper.

\section{APPENDIX E: VISUALIZATION OF THE FIRST SCENARIO FOR THE EXPERIMENTAL SIGNATURE}

In the first experimental scenario, the electrons in the lower band are either in a superconducting $\left(g_{23}<0\right)$ or in a metallic state $\left(g_{23}=0\right)$. The energy difference of the two bands exceeds the Fermi energy, $E_{0}>E_{F}$. Here, we predict that the trimer state can be detected as an excited state. To observe that, one can use either a probe or pump pulse. By optically probing this state one can observe the trimer state as an in-gap resonance peak at the trimer binding energy, $E_{\text {trimer }}$, below the empty band; see Fig. 6 . We predict that the three-body bound state broadens the resonance peak. By optically pumping electrons from the lower band to the upper empty band, we also predict that a trimer state will be formed, if the time scales do not exceed the relaxation rate from the upper band. Figure 6 represents a visualization of the first 

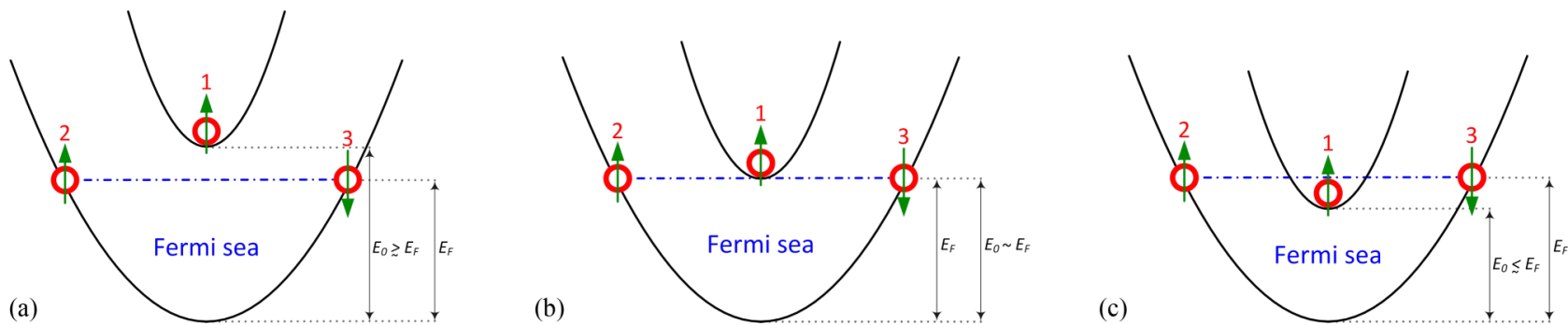

FIG. 7. Sketch of the second scenario for the experimental signature of the trimer states: (a) the upper band is lowered, $E_{0} \gtrsim E_{F}$, (b) it touches the surface of the Fermi sea in the lower band, $E_{0} \sim E_{F}$, (c) it is lowered into the Fermi sea, $E_{0} \lesssim E_{F}$. In all cases we have assumed that the upper band is very dilute.

scenario for both optically probing the state and optically pumping electrons from the lower band.

\section{APPENDIX F: VISUALIZATION OF THE SECOND SCENARIO FOR THE EXPERIMENTAL SIGNATURE}

In the second scenario, the electrons in the lower band are in a superconducting state and the trimer binding energy is larger than the energy difference of the bands, $E_{\text {trimer }}>E_{0}$. Here, we predict that the trimer state can be detected as the ground state of the system. The upper band is lowered, $E_{0} \gtrsim$ $E_{F}$, see Fig. 7(a), and touches the surface of the Fermi sea, $E_{0} \sim E_{F}$, see Fig. 7(b). In both figures the electrons in the lower band are around the surface of the Fermi sea. The upper band can also be lowered into the Fermi sea of the lower band, $E_{0} \lesssim E_{F}$, see Fig. 7(c), giving rise to new electron pockets. Here, we assume that the upper band is very dilute and the Fermi sea is tuned to formation of the electron pockets. In this case we also predict that the trimer state can be formed as the ground state of the system.
[1] L. N. Cooper, Phys. Rev. 104, 1189 (1956).

[2] J. Bardeen, L. N. Cooper, and J. R. Schrieffer, Phys. Rev. 108, 1175 (1957).

[3] A. A. Abrikosov, L. P. Gorkov, and I. E. Dzyaloshinski, Methods of Quantum Field Theory in Statistical Physics (Dover, New York, 1975).

[4] J. R. Schrieffer, Theory of Superconductivity (Westview, Boulder, Colorado, 1999).

[5] M. Tinkham, Introduction to Superconductivity (Dover, New York, 2004)

[6] N. W. Ashcroft and N. D. Mermin, Solid State Physics (Brooks/Cole, Belmont, CA, 1976).

[7] G. Baym, Lectures on Quantum Mechanics (CRC, New York, 1990), Chap. 8.

[8] A. L. Fetter and J. D. Walecka, Quantum Theory of ManyParticle Systems (Dover, New York, 2003).

[9] E. M. Lifshitz and L. P. Pitaevskii, Statistical Physics (Butterworth-Heinemann, Oxford, 2006), Chap. V.

[10] A. J. Leggett, Quantum Liquids: Bose Condensation and Cooper Pairing in Condensed-Matter Systems (Oxford University Press, New York, 2008).

[11] G. Grosso and G. P. Parravicini, Solid State Physics (Academic, Oxford, 2014), Chap. 18.

[12] V. Efimov, Sov. J. Nucl. Phys. 12, 589 (1971) [Yad. Fiz. 12, 1080 (1970)]

[13] V. Efimov, Phys. Lett. B 33, 563 (1970).

[14] F. Ferlaino and R. Grimm, Physics 3, 9 (2010).

[15] P. Naidon and S. Endo, Rep. Prog. Phys. 80, 056001 (2017).

[16] B. Huang, L. A. Sidorenkov, R. Grimm, and J. M. Hutson, Phys. Rev. Lett. 112, 190401 (2014).
[17] S.-K. Tung, K. Jiménez-García, J. Johansen, C. V. Parker, and C. Chin, Phys. Rev. Lett. 113, 240402 (2014)

[18] R. Pires, J. Ulmanis, S. Häfner, M. Repp, A. Arias, E. D Kuhnle, and M. Weidemüller, Phys. Rev. Lett. 112, 250404 (2014).

[19] M. Kunitski, S. Zeller, J. Voigtsberger, A. Kalinin, L. P. H Schmidt, M. Schöffler, A. Czasch, W. Schöllkopf, R. E. Grisenti, T. Jahnke, D. Blume, and R. Dörner, Science 348, 551 (2015).

[20] I. Gierz, M. Mitrano, H. Bromberger, C. Cacho. R. Chapman, E. Springate, S. Link, U. Starke, B. Sachs, M. Eckstein, T. O. Wehling, M. I. Katsnelson, A. Lichtenstein, and A. Cavalleri, Phys. Rev. Lett. 114, 125503 (2015).

[21] R. Mankowsky, M. Först, and A. Cavalleri, Rep. Prog. Phys. 79, 064503 (2016).

[22] D. Nicoletti and A. Cavalleri, Adv. Opt. Photon. 8, 401 (2016).

[23] J.-i. Okamoto, W. Hu, A. Cavalleri, and L. Mathey, Phys. Rev. B 96, 144505 (2017).

[24] In this paper, momentum is measured in units of the Fermi momentum, $k_{F}$, and energy is measured in units of the Fermi energy, $E_{F}=\hbar^{2} k_{F}^{2} / 2 m_{2}$

[25] By "momentum transfer" we simply mean the difference of the in-state and out-state momenta of a particle; see, e.g., J. R. Taylor, Scattering Theory: The Quantum Theory of Nonrelativistic Collisions (Dover, New York, 2006).

[26] G. V. Skorniakov and K. A. Ter-Martirosian, Sov. Phys. JETP 4, 648 (1957)

[27] We have formulated the problem in three dimensions. For one and two dimensions, we do not expect a qualitative change of the integral Eqs. (6) and (7). 
[28] We recall that the shifted energy $E$ was defined as $E=\mathcal{E}-$ $E_{0}$. Thus, for eigenenergies $\mathcal{E}$ near the threshold $E_{\mathrm{thr}}$, we have $E \sim 2 E_{F}$.

[29] L. N. Trefethen and D. Bau, III, Numerical Linear Algebra (SIAM, Philadelphia, 1997).

[30] V. I. Krylov, Approximate Calculation of Integrals (Dover, New York, 2005).

[31] W. H. Press, S. A. Teukolsky, W. T. Vetterling, and B. P. Flannery, Numerical Recipes: The Art of Scientific Computing (Cambridge University Press, New York, 2007).

[32] Notice that, due to the truncation on each sum described in Appendix C, the two-body continuum appears with finite range.
[33] O. Gunnarsson, Rev. Mod. Phys. 69, 575 (1997).

[34] C. Buzea and T. Yamashita, Supercond. Sci. Technol. 14, R115 (2001).

[35] M. Zachmann, M. D. Croitoru, A. Vagov, V. M. Axt, T. Papenkort, and T. Kuhn, New J. Phys. 15, 055016 (2013).

[36] N. Tsuji and H. Aoki, Phys. Rev. B 92, 064508 (2015).

[37] D. Fausti, R. I. Tobey, N. Dean, S. Kaiser, A. Dienst, M. C. Hoffmann, S. Pyon, T. Takayama, H. Takagi, and A. Cavalleri, Science 331, 189 (2011).

[38] S. Kaiser, C. R. Hunt, D. Nicoletti, W. Hu, I. Gierz, H. Y. Liu, M. Le Tacon, T. Loew, D. Haug, B. Keimer, and A. Cavalleri, Phys. Rev. B 89, 184516 (2014). 